\title{
BMJ Open Study protocol for a randomised controlled trial examining the effect of blood and plasma donation on serum perfluoroalkyl and polyfluoroalkyl substance (PFAS) levels in firefighters
}

\author{
Gabriel Silver (D) , ${ }^{1}$ Yordanka Krastev, ${ }^{1}$ Miriam K Forbes, ${ }^{1}$ Brenton Hamdorf, ${ }^{1}$ \\ Barry Lewis, ${ }^{2}$ Michael Tisbury, ${ }^{2}$ Mark P Taylor, ${ }^{1}$ Robin Gasiorowski ${ }^{1,3}$
}

To cite: Silver G, Krastev Y, Forbes MK, et al. Study protocol for a randomised controlled trial examining the effect of blood and plasma donation on serum perfluoroalkyl and polyfluoroalkyl substance (PFAS) levels in firefighters. BMJ Open 2021;11:e044833. doi:10.1136/ bmjopen-2020-044833

- Prepublication history for this paper is available online. To view these files, please visit the journal online (http://dx.doi org/10.1136/bmjopen-2020044833).

Received 15 September 2020

Revised 02 March 2021

Accepted 15 April 2021

A Check for updates

C Author(s) (or their employer(s)) 2021. Re-use permitted under CC BY-NC. No commercial re-use. See rights and permissions. Published by BMJ.

${ }^{1}$ Department of Clinical

Medicine, Macquarie University, Sydney, New South Wales,

Australia

${ }^{2}$ Metropolitan Fire Brigade/Fire Rescue Victoria, Melbourne,

Victoria, Australia

${ }^{3}$ Haematology Department, Concord Repatriation General Hospital, Concord, New South Wales, Australia

Correspondence to

Gabriel Silver;

gab.silver@mq.edu.au

\section{ABSTRACT}

Introduction Perfluoroalkyl and polyfluoroalkyl

substances (PFAS) are a diverse group of compounds that have been used in hundreds of industrial applications and consumer products including aqueous film-forming foam (AFFF) for many years. Multiple national and international health and environmental agencies have accepted that PFAS exposures are associated with numerous adverse health effects. Australian firefighters have been shown to have elevated levels of PFAS in their blood, specifically perfluorooctane sulfonic acid (PFOS) and perfluorohexane sulfonic acid (PFHXS), due to the historical use of AFFF. While PFAS concentrations decline over time once the source of exposure has been removed, their potential adverse health effects are such that it would be prudent to develop an intervention to lower levels at a faster rate than occurs via natural elimination rates.

Methods and analysis This is a randomised controlled trial of current and former Australian firefighters in the Metropolitan Fire Brigade/Fire Rescue Victoria, and contractors, with previous occupational exposure to PFAS and baseline elevated PFOS levels. The study is investigating whether whole blood donation every 12 weeks or plasma donation every 6 weeks will significantly reduce PFAS levels, compared with a control group. We have used covariate-adaptive randomisation to balance participants' sex and blood PFAS levels between the three groups and would consider a $25 \%$ reduction in serum PFOS and PFHXS levels to be potentially clinically significant after 12 months of whole blood or plasma donation. A secondary analysis of health biomarkers is being made of changes between screening and week 52 in all three groups. Ethics and dissemination This trial has been approved by Macquarie University Human Research Ethics Committee (reference number: 3855), final protocol V.2 dated 12 June 2019. Study results will be disseminated via peer-reviewed publications and presentations at conferences. Trial registration number Australian New Zealand Clinical Trials Registry (ACTRN12619000204145).

\section{INTRODUCTION}

Perfluoroalkyl and polyfluoroalkyl substances (PFAS) are a diverse group of compounds

\section{Strengths and limitations of this study}

- This is the first randomised controlled trial to examine a potential treatment for elevated blood perfluoroalkyl and polyfluoroalkyl substance levels.

- While the intervention is open label, the primary endpoints are based on the quantifiable measures of perfluorooctane sulfonic acid (PFOS) and perfluorohexane sulfonic acid (PFHXS) at baseline and week 52

- All participant interventions and assessments from baseline to week 64 are conducted within the same date window, reducing potential study outcome confounders.

- The trial is powered to detect a $25 \%$ reduction in PFOS and PFHXS levels, but it is not clear what level of PFOS reduction is required to improve health outcomes.

- The participant population is from one workforce, located in Victoria, Australia.

resistant to heat, water and oil. Perfluorohexane sulfonate (PFHxS), perfluorooctane sulfonate (PFOS) and perfluorooctanoic acids (PFOA) are the most prominent and commonly detected PFAS. For many years, they have been used in hundreds of industrial applications and consumer products such as carpeting, apparel, upholstery, food paper wrappings, metal coatings and firefighting foams. Based on water, soil and blood samples from the general population, there is evidence that PFAS persist long term both in the environment and in the body. ${ }^{1}$

The persistence of PFAS chemicals is of concern given the emerging evidence they are associated with adverse effects on health. ${ }^{23}$ The Centers for Disease Control and Prevention reported preliminary evidence that PFAS may affect the developing fetus, decrease 
fertility, increase cholesterol, affect the immune system and increase cancer risk. ${ }^{24}$ Elevated PFAS levels have also been associated with increased liver enzyme levels, ${ }^{5}$ increased cholesterol levels ${ }^{6}$ and thyroid disease. ${ }^{7}$ While the specific long-term health effects of elevated serum PFAS remain the subject of debate, ${ }^{8}$ the German Human Biomonitoring Commission (HBM Commission) has recently set a PFOS level of $5 \mathrm{ng} / \mathrm{mL}$, above which adverse health effects cannot be excluded, and below which the HBM Commission has determined there is no risk of adverse health outcomes. ${ }^{9}$

The literature indicates that longer carbon chain PFAS chemicals, including the primary target compounds of PFOS and PFHxS, have a long half-life in humans and are eliminated slowly. ${ }^{10}{ }^{11}$ PFAS are known to be highly protein bound ${ }^{12}$ with significant levels residing in the plasma of exposed individuals, and a prolonged half-life in humans once the source of exposure has been eliminated-8.5 years for PFHxS, 5.4 years for PFOS and 3.8 years for PFOA. ${ }^{13}$ This compares with a half-life of only days in rodents, ${ }^{14}$ making animal studies of their effects difficult to interpret in a human context. This discrepancy highlights the limitations of animal-based research and the need for high-quality human studies in this area. The majority of research on humans has focused on crosssectional analyses of PFAS exposure levels, and thus has not been able to determine how quickly the compounds are eliminated.

The route of elimination of PFAS is also unclear, as there have been no systematic randomised studies of any procedures to eliminate or reduce PFAS levels in humans. Observational studies have found that menstruating women have lower levels of PFAS than men with equivalent exposure ${ }^{15-17}$ In addition, small cohort studies have suggested that regular phlebotomy may reduce serum PFAS levels. ${ }^{18} 19$ Firefighters who donate blood have been found to have lower PFAS levels than firefighters who do not donate. ${ }^{20}$ Taken together, these findings suggest that regular phlebotomy or plasma donation may be a possible intervention to accelerate elimination of circulating PFAS.

The preliminary evidence for adverse health effects of PFAS indicates the importance of developing an intervention to target elevated PFAS levels. In this study, we hypothesise that regular phlebotomy (ie, removal of whole blood) or plasma removal will be effective in reducing serum PFAS levels. Donation of plasma may be more effective than whole blood donation, given plasma is likely to contain most of the total serum PFAS and plasma donation can safely occur more frequently than whole blood donation.

In a study conducted by Rotander et $a l^{20}$ on 149 firefighters working with aqueous film-forming foam (AFFF) at training facilities in Australia, it was found that the highest levels of PFOS and PFHxS were one order of magnitude higher compared with the general population in Australia and Canada, and that concentrations of PFOS and PFHxS in firefighter's blood were associated with the number of years of exposure to AFFF. Internationally, Leary $e t a l^{21}$ also found that PFAS serum concentrations were higher in US firefighters compared with the general population, by between $18 \%$ and $74 \%$.

This study will examine the efficacy of regular blood or plasma donation for reducing serum PFAS levels in a sample of Australian firefighters, a population that has been previously identified as having elevated PFAS (PFOS and PFHxS) levels in their blood compared with the general population. ${ }^{20}$ The data will also provide opportunities to characterise individuals' natural elimination rates of PFAS.

Secondary outcomes of this study include investigating significant differences in lipid profile (total cholesterol, low-density lipoprotein, high-density lipoprotein and triglycerides), thyroid function tests (thyroid-stimulating hormone, T4, T3), liver function tests (bilirubin, alanine aminotransferase, aspartate aminotransferase, gammaglutamyl transferase and albumin), and renal function tests (electrolytes, urea, creatinine) between groups. Statistical significance will not be a focus in these analyses, as we will be underpowered to correct for multiple comparisons.

\section{METHODS}

\section{Study design}

This study is a randomised, controlled, phase II trial of Metropolitan Fire Brigade (MFB)/Fire Rescue Victoria (FRV) staff in Victoria, Australia with previous occupational exposure to PFAS.

\section{Setting}

Prescreening was conducted remotely via phone and email, while screening procedures and baseline questionnaires were conducted in person at a centrally located general practitioner (GP) practice. Blood and plasma donations are conducted at registered blood networks or at the same centrally located GP practice. Records of dates of donations and any adverse events are collected by the clinical project manager remotely via phone and email. Blood tests after screening are conducted through a network of phlebotomy services.

\section{Eligibility}

The eligibility criteria are listed in box 1 . These were used to prescreen participants via email and phone, before screening procedures were undertaken in person. Potential participants needed to meet all inclusion criteria to be eligible for enrolment into the study. Subjects presenting with any of the exclusion criteria were not eligible for the trial (see box 1).

\section{Randomisation}

After being deemed eligible to participate, participants were randomly assigned to either whole blood donation, plasma donation or observation. Covariate-adaptive randomisation was used to balance participants' sex 


\section{Box 1 Inclusion and exclusion criteria}

\section{Inclusion criteria}

- Current or former Metropolitan Fire Brigade/Fire Rescue Victoria staff or contractors, with 10 or more years of previous occupational exposure to perfluoroalkyl and polyfluoroalkyl substances (PFAS) or with known elevated PFAS levels (perfluorooctane sulfonic acid (PFOS) $\geq 5 \mathrm{ng} / \mathrm{mL}$ ).

- PFOS levels $\geq 5 \mathrm{ng} / \mathrm{mL}$.

- Eligible to donate blood.

- Not donated blood in the past 3 months prior to randomisation.

- Signed and dated informed consent document indicating that the participant has been informed of all pertinent aspects of the study prior to enrolment.

- Willingness and ability to comply with scheduled visits, laboratory tests and other study procedures.

\section{Exclusion criteria}

- Medical contraindication to blood donation.

- Other severe acute or chronic medical or psychiatric condition, or laboratory abnormality that may increase the risk associated with study participation or may interfere with the interpretation of study results, and in the judgement of the investigator would make the participant inappropriate for entry into this study.

- Planned travel or extended leave (eg, $>6$ weeks) that would prevent access to blood donation facilities.

and blood PFAS levels, taken at screening, between the three groups. Randomisation was computer generated and conducted centrally after all participants had completed consent, screening procedures and baseline questionnaire. Randomisation was conducted by one of the investigators (MKF) responsible for the statistical aspects of the study, who is not involved in the data collection or intervention. Results of the randomisation were communicated to the clinical project manager (GS) and principal investigator (PI) (RG) prior to the commencement of the intervention.

\section{The intervention}

The study will investigate whether whole blood or plasma donation alters serum PFAS levels by gathering and evaluating data from a discrete occupational cohort-current and former MFB/FRV staff and contractors who have a history of exposure to PFAS substances. The study will be conducted for a total of 18 months, comprising approximately 3 months screening and randomisation, 12 months' intervention and follow-up 3 months thereafter (see figure 1).

Participants randomised to standard whole blood donation are donating blood at blood collection centres or the doctor's office every 12 weeks. Participants are required to complete the standard blood screening procedures including completion of a confidential health questionnaire to check eligibility and a finger prick blood test is used to check haemoglobin levels. Once eligibility to donate is confirmed, participants donate a unit of whole blood (approximately $470 \mathrm{~mL}$ ), following standard blood donation procedures. Participants randomly assigned to the whole blood donation group are scheduled to provide five whole blood donations between baseline and week 48 of the study.

Participants randomised to standard plasma donation are donating plasma every 6 weeks. Participants are required to complete standard blood screening procedures including completion of a confidential health questionnaire to check eligibility and a finger prick blood test to check the haemoglobin level. Once eligibility to
Screening (pre-intervention) Baseline

End of

intervention Follow up period

\begin{tabular}{|c|c|c|c|c|c|c|c|c|c|c|c|c|c|}
\hline Assessment & $\begin{array}{c}\text { Screening } \\
-28 \text { days }\end{array}$ & $\begin{array}{c}\text { Randomisation } \\
\text { 1-3 months }\end{array}$ & $\begin{array}{l}\text { Day 0, } \\
\text { week } 0\end{array}$ & $\begin{array}{c}6 \\
\text { weeks }\end{array}$ & $\begin{array}{c}12 \\
\text { weeks }\end{array}$ & $\begin{array}{c}18 \\
\text { weeks }\end{array}$ & $\begin{array}{c}24 \\
\text { weeks }\end{array}$ & $\begin{array}{c}30 \\
\text { weeks }\end{array}$ & \begin{tabular}{|c|}
36 \\
weeks
\end{tabular} & $\begin{array}{c}42 \\
\text { weeks }\end{array}$ & 48 weeks & 52 weeks & 64 weeks \\
\hline Informed consent & $x$ & & & & & & & & & & & & \\
\hline $\begin{array}{l}\text { Demographics, } \\
\text { medical history, } \\
\text { concomitant } \\
\text { medication (from } \\
\text { questionnaire and } \\
\text { doctor) }\end{array}$ & $x$ & & & & & & & & & & & & \\
\hline Biochemistry & $x$ & & & & & & & & & & & $x$ & \\
\hline FBC & $x$ & & & & & & & & & & & $x$ & \\
\hline EUC & $x$ & & & & & & & & & & & $\mathrm{X}$ & \\
\hline LFT & $x$ & & & & & & & & & & & $\mathrm{X}$ & \\
\hline TFTs & $x$ & & & & & & & & & & & $\mathrm{X}$ & \\
\hline Lipid profile & $x$ & & & & & & & & & & & $x$ & \\
\hline $\begin{array}{l}\text { PFAS blood } \\
\text { sample }\end{array}$ & $x$ & & $x$ & & & & & & & & & $x$ & $x$ \\
\hline Biobank sample & & & & & & & & & & & & $x$ & \\
\hline Blood donation & & & $\mathrm{x}$ & & $x$ & & $x$ & & $\mathrm{X}$ & & $\mathrm{x}$ & & \\
\hline Plasma donation & & & $x$ & $x$ & $x$ & $x$ & $x$ & $x$ & $x$ & $x$ & $x$ & & \\
\hline
\end{tabular}

Figure 1 Study procedure's timeline, from screening to week 64, for all three groups. EUC, electrolytes, urea, creatinine; FBC, full blood count; LFT, liver function test; PFAS, perfluoroalkyl and polyfluoroalkyl substances; TFTs, thyroid function tests. 
donate is confirmed, participants then donate plasma (up to $800 \mathrm{~mL}$, depending on height and weight) following standard donation procedures. Participants randomly assigned to the plasma donation group are scheduled to provide a total of nine plasma donations between baseline and week 48 of the study.

The clinical project manager reminds participants when intervention donations are due and requires notification of the date donations are completed as well as any adverse events that may have occurred either during or between donations. If participants report any adverse events, they are followed up by the clinical project manager to establish the details. Participants are directed to their GP or emergency department for any significant issues. The PI reviews and signs off every adverse event report in a timely manner. Any serious advent events are reported back to the study sponsor within 24 hours according to the Guidelines for Good Clinical Practice (GCP) and reviewed by the PI or delegate within 24 hours of knowledge.

\section{Participant recruitment}

The study was promoted to all MFB/FRV staff through in-person presentations, internal email communications, and via study posters and brochures sent to all firefighter stations. Interested MFB/FRV staff registered their interest via email and were prescreened via phone. Those passing prescreening received the patient information and consent form via email and were invited to attend a screening visit at a centrally located GP office. It was here that medically qualified clinical study personnel explained the trial protocol with participants and answered any questions they may have had. Informed consent was obtained before conducting the initial screening for the study.

Participant screening and consenting took place between 23 May and 23 August 2019. Our target was to recruit a total of 315 participants (105 per group) to be randomised into the study. At the end of the screening period, 333 participants had been screened and consented, with 285 deemed eligible after screening PFOS level results were analysed.

\section{Data collection procedures}

Following participant consent to take part in the study at screening, they were asked to complete a questionnaire comprising contact details, demographics, lifestyle information, donation history, medical history, medication listing and occupational history.

Participants also provided a screening PFAS blood sample. The blood samples were analysed by Envirolab Services Pty Ltd for 28 different PFAS compounds. The study protocol required that participant enrolment was based on PFOS levels in blood given that this was a wellestablished component of AFFF and has been shown previously to be a proven marker of occupational AFFF exposure. $^{2}$

On receipt of the participant PFAS serum concentrations, final eligibility was judged according to whether PFOS levels were above or equal to $5 \mathrm{ng} / \mathrm{mL}$.
The study protocol is designed with PFAS blood tests performed at screening, baseline, week 52 and week 64 . All blood tests conducted during the study are analysed at a National Association of Testing Authorities-certified facility. Screening and baseline PFAS blood tests are designed to establish natural underlying clearance rates of PFAS in the participants.

Week 52 PFAS blood test is the primary endpoint for comparison with the baseline PFAS blood test to assess the impact of two interventions (blood and plasma donation) and whether the levels of circulating PFAS have been reduced.

A follow-up PFAS blood test at week 64 will allow comparison with individuals' pre-intervention clearance rates to quantify whether PFAS levels continue to decline at the same rate or rebound after cessation of intervention at week 48. There is a possibility that the intervention will only facilitate the reduction of circulating PFAS and will not impact PFAS stored in other tissues, which may cause an increase in circulating PFAS levels when the intervention ends.

Participants will not have access to their PFAS blood test results until after the study is completed. This will reduce the impact of any confounding variables such as MFB/FRV staff in the control group donating blood if they find they have high PFAS levels, or those with low levels missing donation appointments. The PFAS blood test results of samples taken at screening and week 52 will be sent to the participants' GP at the end of the study (as consented by the participant), accompanied by a letter from the PI and a PFAS blood result fact sheet.

The study also includes biochemistry analysis. Biochemistry samples are taken at screening and week 52 and assess the following biomarkers: urea and electrolytes, liver function, lipid levels, full blood examination and thyroid test. These tests may also provide initial insight into early adverse effects of PFAS exposure at different levels.

The study is projected to complete data collection, with the collection of week 64 PFAS blood tests from the last participant, by 28 February 2021.

\section{Statistical methods}

We have planned our analyses based on intention-to-treat and will use multiple imputation to handle missing data. G*Power was used for a priori power analyses. We would consider a 25\% reduction in serum PFOS and PFHxS levels to be potentially clinically significant after 12 months of plasma or whole blood donation. Using a dependent sample t-test based on the mean serum levels of PFHxS and PFOS found in 149 Australian firefighters, ${ }^{2}$ and a correlation between the assessments at baseline and 12 months (post-test) of $\mathrm{r}=0.6$, this corresponds to a standardised effect size of mean difference $d_{z} \geq 0.3$. Each arm (whole blood donation, plasma donation and observation) requires 94 participants to have $90 \%$ power to detect a $25 \%$ reduction in PFOS and PFHxS levels within the group. The recruited 


\section{Consort Flow Diagram \\ PFAS Clinical Trial}

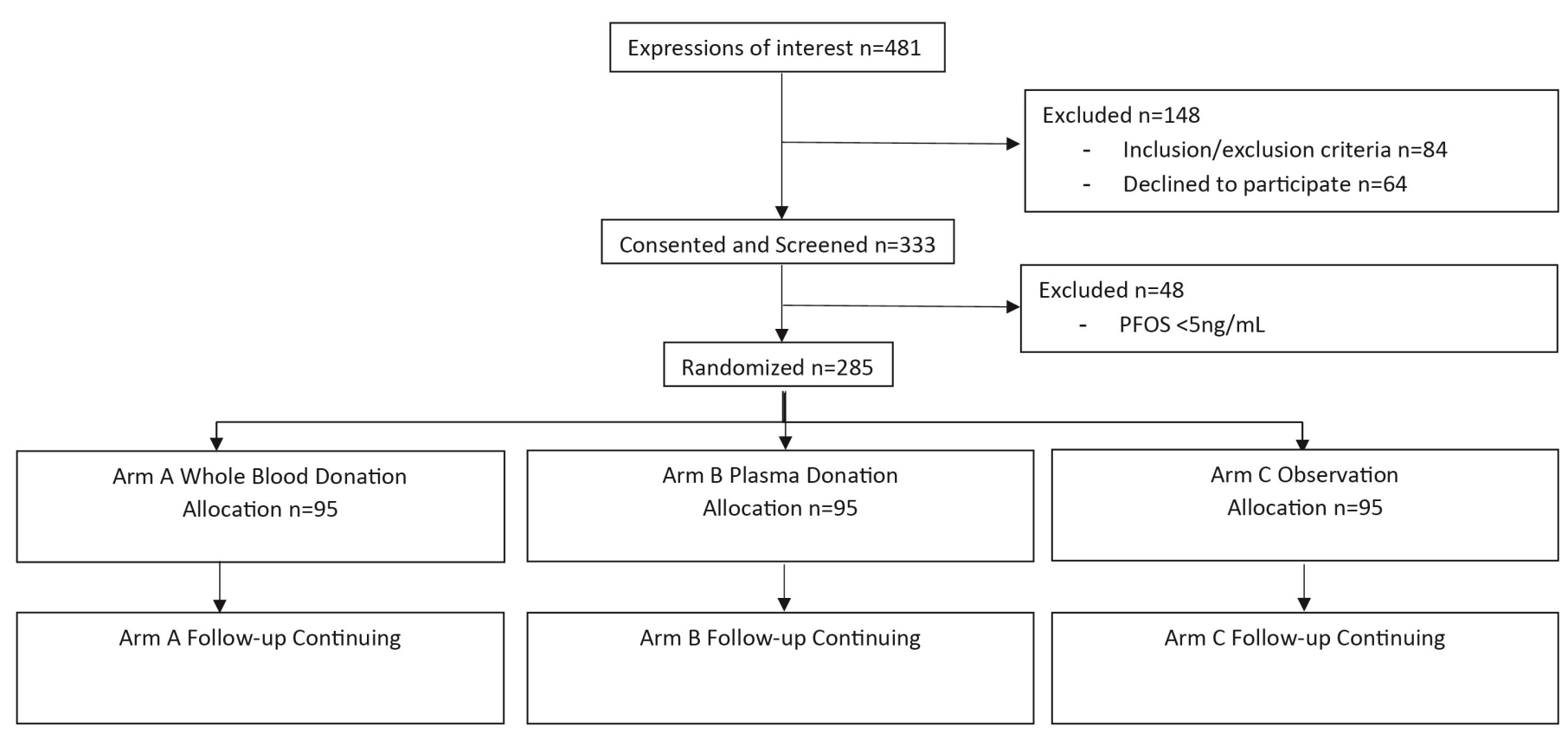

Figure 2 CONSORT flow diagram from prescreening to current follow-up of participants. CONSORT, Consolidated Standards of Reporting Trials; PFAS, perfluoroalkyl and polyfluoroalkyl substances; PFOS, perfluorooctane sulfonic acid.

sample of 95 participants per group (see figure 2) achieves power of $91 \%$ to detect the same effect size.

To test whether plasma donation reduces serum PFOS and PFHxS levels at a faster rate than whole blood donation, our a priori power analyses found that each group required 105 participants for $90 \%$ power to detect a conventional small effect size (partial $\eta^{2}=0.01$ ) difference between the groups from pre-test to post-test in a repeated measures analysis of variance framework including a within-between interaction. The actual sample size of 95 participants per group (see figure 2) achieves power of $87 \%$ to detect the same effect size (and $90 \%$ power to detect partial $\eta^{2}=0.011$ ). Similarly, to further compare the efficacy of plasma donation and whole blood donation to the observation only group (control)-after a conservative Bonferroni correction for multiple testing to minimise type II error $(\alpha=0.017)$ - the sample of 95 participants per group provides $75 \%$ power to detect the same small effect size (partial $\eta^{2}=0.01$; and $90 \%$ power to detect partial $\eta^{2}=0.014$ ).

\section{Ethics and dissemination}

The study has been approved by the Macquarie University Human Research Ethics Committee (HREC) before commencing (reference number: 3855).

Informed consent meets the requirements of the latest revision of the National Statement on Ethical Conduct in Human Research (2018) and the Guidelines for GCP in Australia. The study was explained to each potential participant by a trained clinician, with signed informed consent obtained for all participants included in the study before any study procedures were performed. Participants were given a printed copy of the participant's information sheet and consent form.

All participants have been informed of the aims of the study, the possible adverse events, the procedures and possible hazards to which they might be exposed. They have been informed as to the strict confidentiality of their participant data, but that their medical records may be reviewed for clinical research purposes by authorised individuals other than their treating physician.

It has been emphasised that participation is voluntary and that the participant is free to refuse further participation in the study at any time without repercussion.

If any important protocol amendments, such as changes to eligibility criteria, outcomes or analyses, occur after participant consent, these shall be communicated to all relevant parties (eg, investigators, HREC/institutional review boards, trial participants, trial registries, journals, regulators) immediately and reconsent obtained as appropriate.

\section{Confidentiality and monitoring}

Every participant has been given an individual numerical study number, which is used on all pathology forms and in the online study database. The master log containing the data key of the participant identifiable information and allocated study number is accessible only by the clinical project manager and PI. All study documents in paper form are kept in a locked secure cabinet in the office of the clinical project manager in Victoria, Australia, with all electronic study information stored in the password-protected 
online study Research Electronic Data Capture (REDCap) Database. All study documents will be archived in a secure location after the end of the trial.

The data manually entered into the REDCap Database will be monitored by a research professional hired by Macquarie University, but not involved in the study. One hundred per cent of endpoint data shall be monitored, with $10 \%$ of all other data also monitored for accuracy. This shall occur after all data collection has taken place. The Data Management Committee is comprised of the same members as the Project Management Committee.

\section{Patient and public involvement}

MFB/FRV staff have been involved in the design and implementation of this clinical trial. However, the final protocol and oversight of the trial is the responsibility of Macquarie University.

\section{Project management}

The study is led by a Project Management Committee which meets every 2 weeks and that comprises the Macquarie University-affiliated authors of this paper. Those being: Clinical Project Manager Gabriel Silver, MHA, gab.silver@ mq.edu.au; Yordanka Krastev, PhD, yordanka.krastev@ mq.edu.au; Miriam K Forbes, PhD, miri.forbes@mq.edu. au; Mark P Taylor, PhD, mark.taylor@mq.edu.au; Brenton Hamdorf, PhD, brenton.hamdorf@mq.edu.au; and Robin Gasiorowski, PhD, robin.gasiorowski@mq.edu.au.

The Project Management Committee meets with the sponsor's MFB/FRV PFAS Fire Fighter Study Project Management Group every 2 weeks to ensure sponsor governance, oversight, and to discuss progress and any issues that require resolution. MFB/FRV PFAS Fire Fighter Study Project Management Group includes Michael Tisbury ( michael.tisbury@frv.vic.gov.au) and Barry Lewis (barry. lewis@frv.vic.gov.au).

The study also has an Advisory Panel made up of international experts in PFAS and clinical trials. This panel meets quarterly and reviews all data and adverse events for trends, focusing on patient safety and data integrity.

\section{Trial status}

The trial is underway with screening, recruitment and baseline visits complete. The intervention has commenced, and follow-up is continuing. The trial is currently projected to complete the last data collection point by April, 2021.

\section{Dissemination}

As the first study of its kind, study findings will be published in a peer-reviewed journal as soon as possible after data analysis and shall directly inform interventional strategies to reduce the potential health risks associated with PFAS exposure.

\section{Correction notice Figure 2 has been corrected.}

Acknowledgements The Advisory Panel was chaired by Gabriel Silver and comprised of Mark P Taylor, Merrole Cole-Sinclair of St Vincent's Hospital, Victoria, Australia; Bruce P Lanphear of Faculty of Health Sciences, Simon Fraser University, Vancouver, British Columbia, Canada; Roger A Klein of Cambridge, UK and Christian Regenhard Center for Emergency Response Studies (RaCERS) CUNY New York; Nigel Holmes of Queensland Department of Environment and Science (DES), Queensland Australia; Michael Tisbury (observer); and Robin Gasiorowski. Drs Brendan Crockett, Julian Foo, and Munita Singh consented and screened participants at the centrally located General Practitioner (GP) Practice St Vincent's Medical Centre, Australian Catholic University campus, Fitzroy, Victoria, Australia. St Vincent's Hospital Melbourne GP Clinics network-facilitated phlebotomy services. Envirolab Services Pty Ltd facilitated all PFAS testing.

Contributors MT and MPT conceived the study. BH, YK, MKF and RG designed the initial study protocol and prepared the initial ethics submission. GS is the clinical project manager, contributed to the final protocol design and wrote the initial draft of this paper. BL and MT represented the sponsor MFB/FRV and were consulted during the protocol design. All authors have been involved in reviewing and editing the manuscript, and read and approved the final draft.

Funding This study was funded in full by the sponsor: Metropolitan Fire Brigade/ Fire Rescue Victoria, Victoria's professional Fire Service and a statutory authority. Contact: michael.tisbury@frv.vic.gov.au; barry.lewis@frv.vic.gov.au.

Competing interests None declared.

Patient and public involvement Patients and/or the public were involved in the design, or conduct, or reporting, or dissemination plans of this research. Refer to the Methods section for further details.

Patient consent for publication Not required.

Provenance and peer review Not commissioned; externally peer reviewed.

Open access This is an open access article distributed in accordance with the Creative Commons Attribution Non Commercial (CC BY-NC 4.0) license, which permits others to distribute, remix, adapt, build upon this work non-commercially, and license their derivative works on different terms, provided the original work is properly cited, appropriate credit is given, any changes made indicated, and the use is non-commercial. See: http://creativecommons.org/licenses/by-nc/4.0/.

ORCID iD

Gabriel Silver http://orcid.org/0000-0003-3249-1062

\section{REFERENCES}

1 United States Environmental Protection Agency (USEPA). Research on per- and Polyfluoroalkyl substances (PFAS). United States environmental protection agency, 2018. Available: https://www.epa. gov/chemical-research/research-and-polyfluoroalkyl-substancespfas

2 Agency for Toxic Substances and Disease Registry (ATSDR). Toxicological profile for perfluoroalkyls. draft for public comment (June 2018). US Department of Health and Human Services, Agency for Toxic Substances and Disease Registry (ASTDR) (\#CS274127-A), 2018. Available: https://www.atsdr.cdc.gov/toxprofiles/tp.asp?id= 1117\&tid=237

3 Rappazzo KM, Coffman E, Hines EP. Exposure to perfluorinated alkyl substances and health outcomes in children: a systematic review of the epidemiologic literature. Int J Environ Res Public Health 2017; 14:691.

4 International Agency for Research on Cancer (IARC). Perfluorooctanic acid. International agency for research on cancer monograph, 2018. Available: https://monographs.iarc.fr/wp-content/ uploads/2018/06/mono110-01.pdf

5 Gleason JA, Post GB, Fagliano JA. Associations of perfluorinated chemical serum concentrations and biomarkers of liver function and uric acid in the US population (NHANES), 2007-2010. Environ Res 2015;136:8-14.

6 Steenland K, Tinker S, Frisbee S, et al. Association of perfluorooctanoic acid and perfluorooctane sulfonate with serum lipids among adults living near a chemical plant. Am J Epidemiol 2009:170:1268-78.

7 Knox SS, Jackson T, Frisbee SJ, et al. Perfluorocarbon exposure, gender and thyroid function in the $\mathrm{C} 8$ health project. J Toxicol Sci 2011;36:403-10.

8 Kirk M, Smurthwaite K, Bräunig J. The PFAS health study: systematic literature review. Canberra: The Australian National University, 2018.

9 Apel P, Angerer J, Wilhelm M, et al. New HBM values for emerging substances, inventory of reference and HBM values in force, and working principles of the German human biomonitoring Commission. Int J Hyg Environ Health 2017;220:152-66. 
10 Olsen GW, Mair DC, Lange CC, et al. Per- and polyfluoroalkyl substances (PFAS) in American red cross adult blood donors, 20002015. Environ Res 2017; 157:87-95

11 Zhang Y, Beesoon S, Zhu L, et al. Biomonitoring of perfluoroalkyl acids in human urine and estimates of biological half-life. Environ Sci Technol 2013;47:10619-27.

12 Jones PD, Hu W, De Coen W, et al. Binding of perfluorinated fatty acids to serum proteins. Environ Toxicol Chem 2003;22:2639-49.

13 Olsen GW, Burris JM, Ehresman DJ, et al. Half-life of serum elimination of perfluorooctanesulfonate,perfluorohexanesulfonate, and perfluorooctanoate in retired fluorochemical production workers. Environ Health Perspect 2007;115:1298-305.

14 Kim S-J, Heo S-H, Lee D-S, et al. Gender differences in pharmacokinetics and tissue distribution of 3 perfluoroalkyl and polyfluoroalkyl substances in rats. Food Chem Toxicol 2016;97:243-55.

15 Wong F, MacLeod M, Mueller JF, et al. Enhanced elimination of perfluorooctane sulfonic acid by menstruating women: evidence from population-based pharmacokinetic modeling. Environ Sci Technol 2014;48:8807-14.
16 Kato K, Wong L-Y, Jia LT, et al. Trends in exposure to polyfluoroalkyl chemicals in the U.S. population: 1999-2008. Environ Sci Technol 2011;45:8037-45.

17 Toms L-ML, Thompson J, Rotander A, et al. Decline in perfluorooctane sulfonate and perfluorooctanoate serum concentrations in an Australian population from 2002 to 2011. Environ Int 2014;71:74-80.

18 Genuis SJ, Liu Y, Genuis QIT, et al. Phlebotomy treatment for elimination of perfluoroalkyl acids in a highly exposed family: a retrospective case-series. PLoS One 2014;9:e114295.

19 Lorber M, Eaglesham GE, Hobson P, et al. The effect of ongoing blood loss on human serum concentrations of perfluorinated acids. Chemosphere 2015;118:170-7.

20 Rotander A, Toms L-ML, Aylward L, et al. Elevated levels of PFOS and $\mathrm{PFH}$ XS in firefighters exposed to aqueous film forming foam (AFFF). Environ Int 2015;82:28-34.

21 Leary DB, Takazawa M, Kannan K, et al. Perfluoroalkyl substances and metabolic syndrome in firefighters: a pilot study. $J$ Occup Environ Med 2020;62:52-7. 